\title{
BLENDED LEARNING TECHNOLOGIES IN PRODUCT DESIGN EDUCATION
}

\author{
Dr Timothy WHITEHEAD, Dr Lyndon BUCK and Jonathan HEWITT \\ Aston University, UK
}

\begin{abstract}
One of the notable consequences of the COVID-19 pandemic has been a radical shift to blended learning across education settings, including HE. Blended learning has risen to prominence in the last year as students and tutors have been forced to adopt and adapt to new ways of working. Creative subjects such as design which rely on studio practice, peer-peer learning, and hands-on material experimentation have been challenged through adoption of these changes. This paper explores the short-term changes which have been made and assesses the impact for the near and long term future of design pedagogy. The study focuses on three contrasting accredited UK BSc product design courses; (1) top 40 rank HEI (2) bottom 20 rank HEI (3) online HEI. The authors reviewed course materials, suitability and adaptability for online teaching and student outputs from the last 3 years, including the use of online environments and digital prototyping to try and establish virtual design studios. Feedback from students and tutors is included and evaluated. The contrast between intrinsic and extrinsic motivations in students from the 3 different teaching institutions is discussed along with examples of learning and teaching activities and their efficacy. The findings conclude that while innovative teaching methods are evident, there is a lack of design iteration and innovation, peer-peer learning and practised understanding of form and proportion. While new teaching methods and techniques show promise, the present need to maintain physical studio and learning spaces and to enhance the culture of practical, physical working is noted.
\end{abstract}

Keywords: Blended learning, studio practice, virtual design studios, digital prototyping

\section{INTRODUCTION}

The Covid-19 lockdown forced 1.6 billion children and students [1] out of schools and universities worldwide, pushing many onto a steep edtech learning curve. The move from physical to distanced models has challenged many educators [2] and acutely affected design and engineering, where the removal of studios, laboratories, workshops, and shared learning spaces have forced many to rethink the design and delivery of their learning, "going from denial to acceptance" (Brown, 2020). For some, the edtech boom is long overdue. Andreas Schleicher, head of education at the Organisation for Economic Co-operation and Development (OECD), described the pandemic as "a great moment" for learning [3].

\subsection{Blended learning}

In May 2020, New York governor Andrew Cuomo publicly questioned why physical classrooms still exist at all, as he announced that former Google CEO Eric Schmidt and Bill Gates would help rethink education in the state [4]. Critics like Naomi Klein say tech giants were quick to see Covid-19 as an opportunity to accelerate their education ambitions [5]. "In moments of crisis, people are willing to hand over a great deal of power to anyone who claims to have a magic cure" [6]. In June, Microsoft published a position paper on Remote to Hybrid Learning called Education Reimagined. "The fallout from Covid19, continuing advances in digital technology, and intensifying pent-up demand for student-centred learning have combined to present an unprecedented opportunity to transform education across whole systems." [7]. But will we continue this digitally enhanced approach, post-pandemic? Covid has given an impetus to adopt more functional digital edtech tools, comments Hannah Owen, of the Nesta innovation foundation, and 'it's likely, and optimal, that we'll move to blended models, where remote and digital platforms support in-person classroom teaching and minimise teacher workload." [8]. But there are challenges, such as assessing which students have understood a question "that's a lot harder to do with video conferencing software or digital worksheets" [9] and could be more time consuming, not less. Another area which could rise to prominence is the use digital tools to identify who needs help 
and if used well, analytics and big data could help engage students and individually help tailor input. Some found that virtual learning environments helped students engage better, gain more control over their learning, work in ways that suit their needs, and minimise anxieties by engaging via the chat [8].

\subsection{Virtual studios}

Critical to a blended learning approach is not only to covey learning material but also to support peerto-peer learning, which is typically practiced in a studio environment. A common concern of blended learning for design education is that quality of student output will deteriorate if students are unable to compare work during formal or informal critiques, group activities and group learning. To overcome this, virtual studios have become prominent in the shift to online learning. One of the first virtual studios Open Design Studio (ODS) has been created by the Open University (OU) in 2010. One of the key factors for success is the ability to view other students' work, thus demonstrating a stronger (or equal) correlation of student success compared to any other behaviour measured [10]. Students on OU design courses can choose to share visual ideas and design solutions with their peers. They can also comment on peer's work, and with around 500 students active on a module at any time, form part of a virtual learning community, giving great feedback potential, more so than in a physical studio. Engaging these feedback and community behaviours is part of a larger set of social learning behaviours that contribute to an 'ecology' of digital studio learning [10]. Viewing other students' work, far from being inactive or passive learning, was found to be an intrinsic motivator and contributed to a positive learning experience, noting that measuring student engagement in this process was important - the issue of 'not leaving a mark'. The shift to remote learning has challenged the use of physical studio environments how can we re-think approaches to design education, evaluate, and learn from this experience and understand how design can adapt to emerging virtual learning environments and future design studios?

\section{METHODOLOGY}

In order to understand the impact of blended learning and studio environments this study focuses on comparing and contrasting the interaction, engagement, technical adoption, and output of 3 accredited UK BSc product design courses; (1) top 40 rank university (2) bottom 20 rank university (3) online provider. This included an analysis of course material, suitability and adaptability for online teaching and final year student outputs over the last 3 years. A particular focus is the adoption and inclusion of online environments and digital prototyping to establish how, and if, virtual design studio spaces are utilised. The contrast between intrinsic and extrinsic motivations of students is discussed along with examples of learning and teaching activities and their efficacy. The results are analysed to draw out key themes about the use, uptake, and output of student work in a virtual studio environment. The findings have been split into four themes across each course: technology uptake, student engagement, quality of design output and future industry demands. These four areas demonstrate critical elements to ascertain the effectiveness of approaches and inform recommendations for blended studio practice moving forward.

\section{TECHNOLOGY}

Advances in technology have offered additional tools and experiences to enrich the virtual element of blended learning, beyond traditional OBS. Critically for design, some web-based tools assist in creative activities such as group ideation. Platforms such as 'Mural' (Figure 1) and 'Miro' offer a space where groups meet and work virtually and collaboratively and enable functions encouraging engagement, such as voting, templates to stimulate thinking, and icons and sketching tools to enhance spontaneity. These tools are important as they lower the point of entry and enable students to engage with a stream of visual content which resonates with those who naturally engage with social media platforms. However, these technologies and remote collaboration lack the social dynamic of a physical environment. Currently, it is a very different experience to be working over a digital platform than it is to be around a physical table. In all 3 institutions this was identified as a continuing challenge. Group learning is different, traditional points of discovery or clarity are hard to detect when online. Therefore, consideration of this is important when working in a blended environment, the application and facilitation of physical workshop activities into a virtual space is very different to that of a physical one. Students often note how group exercises are reliant on one or two individuals doing much of the work or talking, rather than a team effort. Physical engagement can often help to alleviate that problem, via intervention and observation of participants. Serendipity, as a design tool, is not as easy to encourage or witness in a 
virtual space. This in turn means that critiques of work become far more considered due to the mindset of the participants being more focused when posting comments rather than verbalising them. Tone of voice, cadence and physical expression are not apparent and count for a lot in those scenarios. In addition, it was highlighted that building trust becomes difficult due to the anonymity of virtual spaces, it is hard for participants to truly understand each other at distance, which can make the difference between a student engaging with subject matter or not. We are inherently social, and this dynamic is a rich element in current models of learning and communication. The blended approach allows for fewer opportunities for physical interaction, which poses the question - how can they be effectively replaced? How are we changing our social habits and design methods in a learning landscape that is becoming more virtual?

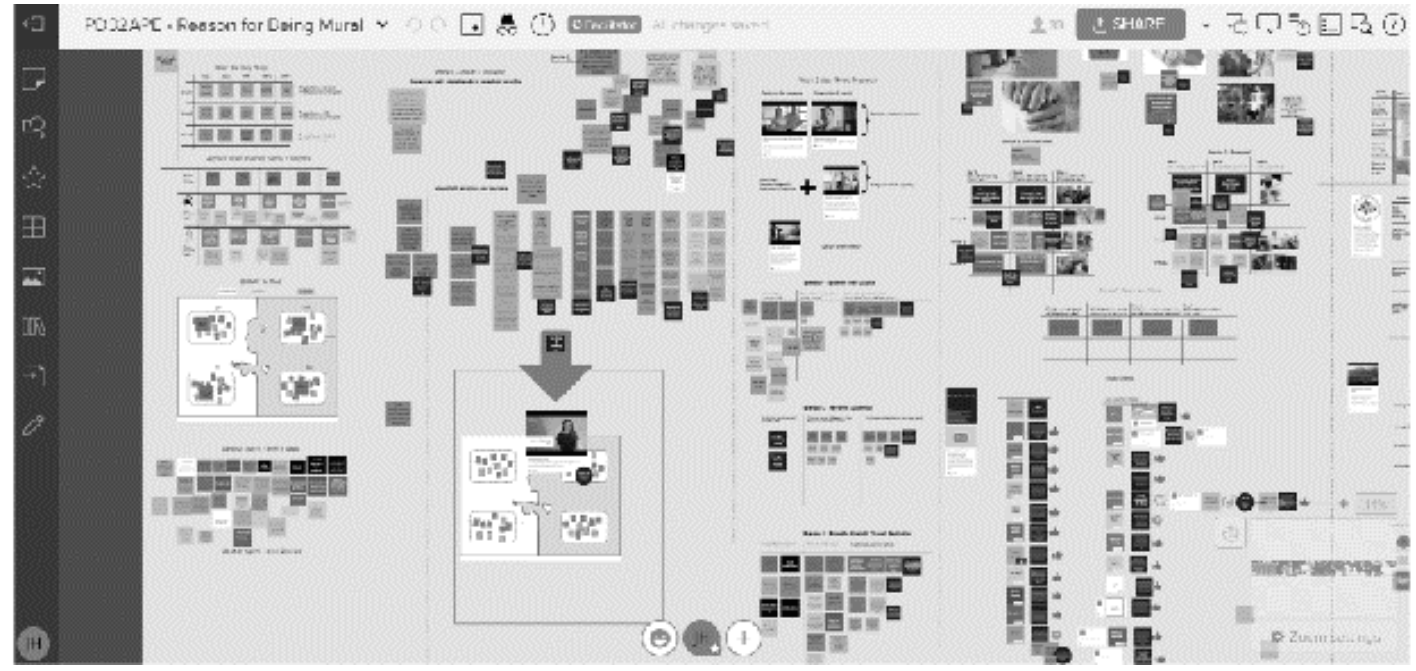

Figure 1. Second year collaborative design group work on Mural

\section{ENGAGEMENT}

Student engagement and collaboration is key to successfully achieving learning objectives and enriching the education experience. In each of the 3 institutions studied it was identified that participation can manifest itself in many ways from passive observation and listening through to active posting and contribution. Engagement as a metric is harder to measure in a digital space, it is easier for students to 'drop off the map' [9]. Feedback from group exercises indicated that this type of activity can be alienating to some (due to others taking a lead) as well as being too easy to sit back and watch rather than engage. A key finding was that we must consider character types and how content can be structured to sustain engagement and adjusted to ensure an appropriate barrier to entry when it comes to participation. An example of this was identified by one institution using Mural to discuss the feelings and emotions portrayed by contrasting designed objects. Students posted keywords on individually coloured notes. This low barrier to entry facilitated effective engagement with the activity, which then ramped up to empowering students to critique their own and others work in the platform (Figure 2).

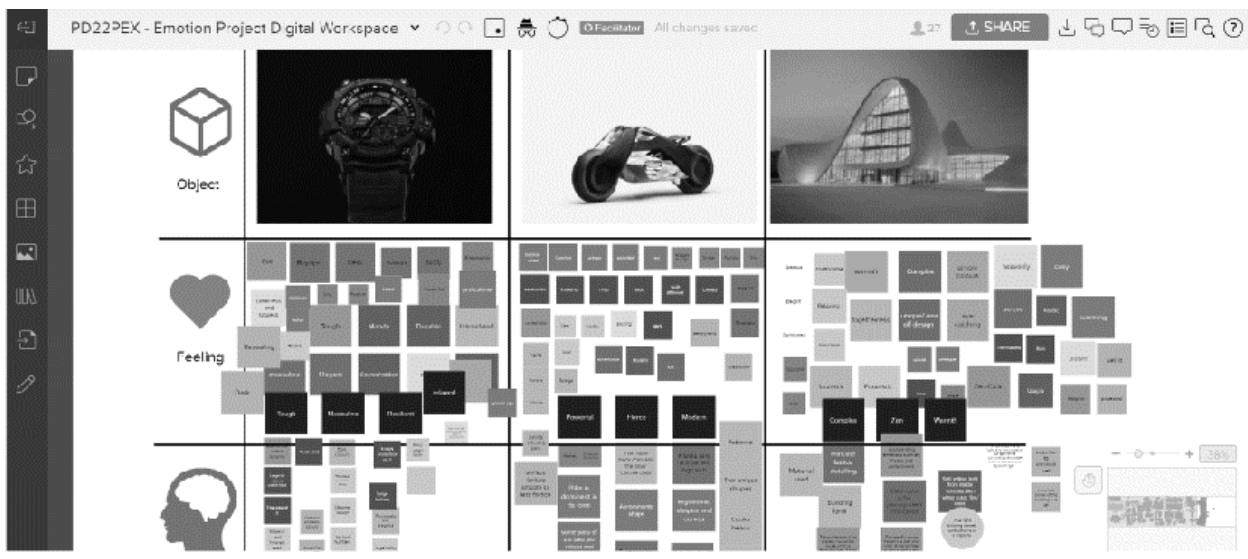


To encourage engagement, regular feedback on work generates a feeling of progress and flow similar to engagement on social media. Collaboration spaces offer a $24 / 7$ opportunity to discuss work, with students witnessing an emerging body of work which often has a positive influence. Creativity can be seen and shared, providing inspiration moving forward. Virtual spaces allow for visual exploration using parallels and creative approaches (metaphors) to express new thinking and approaches. Fresh content helps maintain momentum, weekly updates of new videos, podcasts and exercises add to understanding. Material helps to stimulate debate and interaction, fuelling conversations, empowering collaboration. Learning in isolation is one route students can take from virtual spaces, however the richness and reward from partnerships of group and peer discussion offer valuable context and perspectives. Encouragement of positive behaviours and attitudes helps nurture this mode of working with those new to it. Supporting dialogue, visual stimuli (imagery and emojis) are useful and drive a familiarity to the virtual medium. It is useful to clarify etiquette with regard to engagement. Parallels can be drawn with social media platforms; this can shape a mindset, but posts should be monitored.

\section{STUDENT OUTPUT}

Critical tests for digital studio environments are quality of student outputs, attainment and evidence of design thinking, awareness of visual design language and balance between product form, function, and end user ergonomics. It was identified that in a virtual online environment there is a greater focus on design thinking and developing and critiquing ideas. However, it is evident that there is a loss of physical prototyping and testing. Although it is evident that some students will photograph low-fidelity models and upload them to gain feedback, there are a number of steps to this process. Students often feel that a low fidelity prototype is not of sufficient quality to publish on a virtual platform. For the Instagram generation, pictures are routinely filtered and edited before uploaded. There may be a fear that poor quality images and work will stay online forever, and this could damage the student's reputation (Figure 3). These issues can be present in a traditional studio space, but when students show their work it is typically on the table/wall for a short period of time and then removed quickly to improve. This presents a contrast on the one hand students enjoy brainstorming and linking to new product ideas but are less keen to have a visual critique. It was identified that the uptake and engagement of this activity was significantly lower, but by removing low fidelity work directly after critiques this effect was reduced.

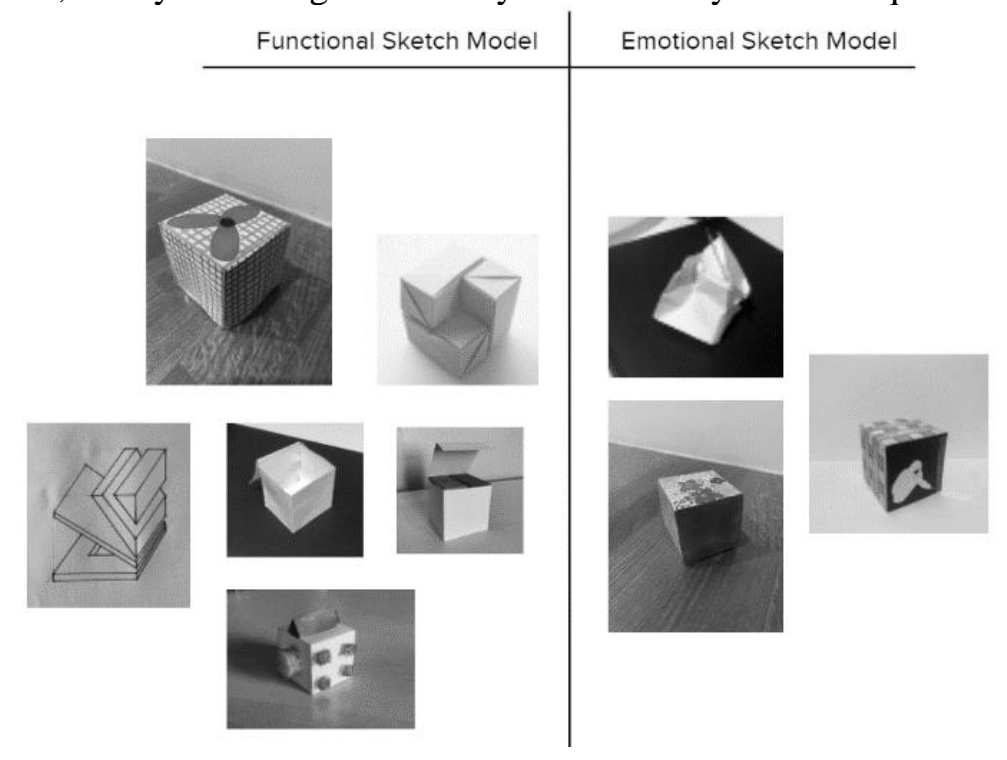

Figure 3. Low fidelity sketch models presented in Mural

The final design output, typically at an end of year assessment, highlights that most students produce less convincing design solutions, which are often more conservative, safe and lack the depth of development which is seen in a face-face studio. When looking at purely digital outputs it was expected that free from traditional prototyping constraints students would show greater levels of experimentation and innovation, yet the opposite happened. Instead of pushing the boundaries it was evident that students placed their own virtual boundaries, tried to anticipate the comments of workshop technicians, and 
instead held themselves back. An example of this was seen in outputs in 2020 (Figure 4) compared with the start of 2021 in which face-face studio practice happened (Figure 5). It is evident that that the depth of development, innovation and sensitivity to form is lost when the 3 best students are compared. This is reported to be due to a lack of face-to-face feedback, physical prototyping and testing available online.
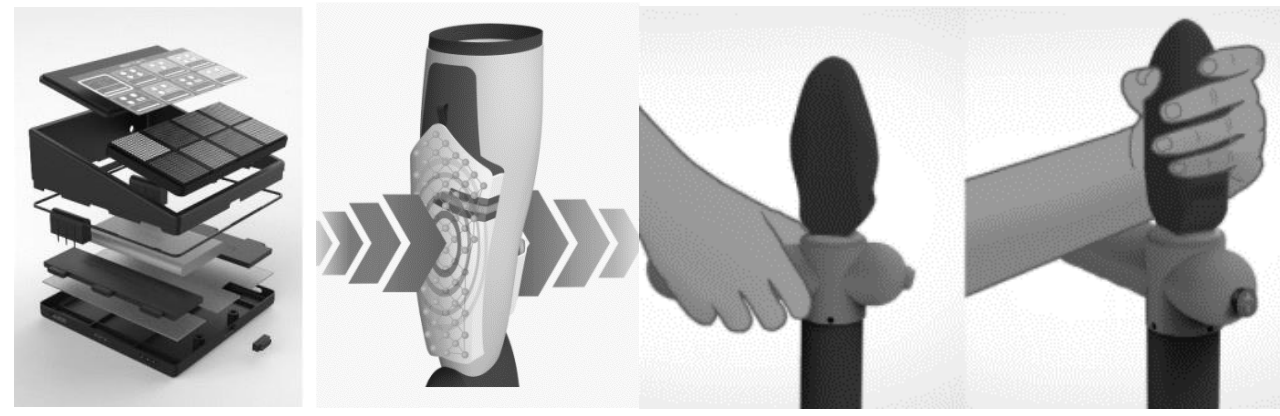

Figure 4. Highest achieving students in remote studio environment

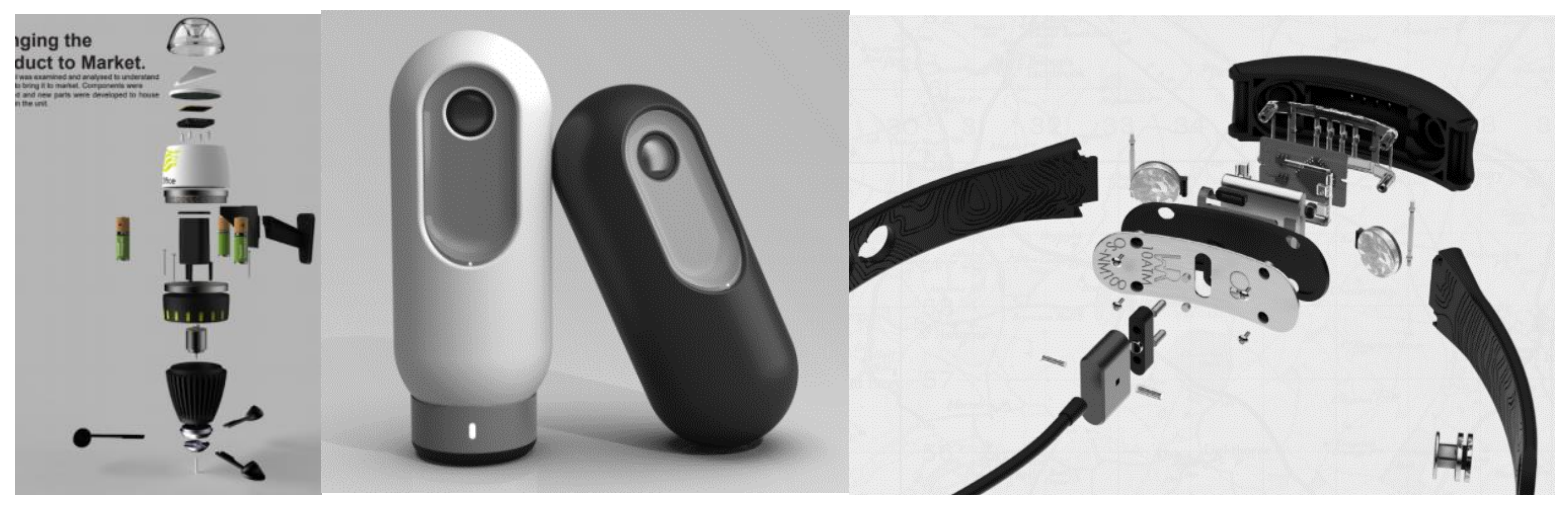

Figure 5. Highest achieving students in physical design studio

\section{INDUSTRY EXPECTATIONS}

Blended learning offers opportunities to collaborate beyond the constraints of lecture theatres or traditional classroom environments. The ability to incorporate external collaborators into modules is enhanced through the ability to hold virtual seminars, with geography no longer a barrier. This way of working shares parallels with industry during the pandemic, satellite teams and virtual collaboration have become prevalent out of necessity. This allows for companies to recruit globally, without the need for relocation, tapping into a broader talent source of talent. Within design this can enrich thinking, offering perspectives from a mixture of different references and cultures avoiding 'group think' from local teams. Looking forward to employment, blended learning delivers an opportunity whereby students develop the mindset to become more independent learners. Depending on the module and its delivery it is possible to steer the student to content creators, platforms and topics allowing them to leverage large amounts of information available online. This skill of following others, seeking views, and networking within digital communities can prove to be an important and enriching skill when in employment. Video presentations, use of social channels and leveraging the digital relationships of oneto-many are becoming more prevalent. Covid 19 has accelerated remote work and virtual collaboration.

\section{CONCLUSIONS}

In a post-COVID world there is an increasing challenge to justify physical learning environments and balance the adoption of digital tools and virtual learning spaces. This paper goes some way to further this discussion in relation to design education. The findings demonstrate that there are advantages to remote working and digital studios, but this comes at a cost to human interaction, degradation of physical prototyping and mixed levels of engagement. Virtual studios have been shown to work in the initial stages of design projects, to generate ideas, capture inspirational content and to develop team thinking. However, this is reliant on each student being actively engaged during the whole process. The design outputs suggests that there is a fundamental need to maintain physical studios and practical prototyping. 
It is easy to slip into a mindset whereby direct comparisons are made between classical and blended learning environments. The objectives of imparting knowledge and encouraging growth are common. However due to humanistic elements such as group dynamics, expression, and interaction the individual experience can be very different. For the blended approach to be successful we must consider the medium carefully, not to fundamentally change the learning objectives but to impact the method of delivery. Students are being asked to learn in a way that few have experienced previously. In easing this transition the authors are piloting several initiatives and approaches to support the learning experience:

- Encourage and celebrate online collaborative behaviour, including online participation in exercises, posting of work and progress, commenting and supportive critiques of other students

- Leveraging benefits of online working by hosting guest speakers and presentations, integrating content from online platforms and channels into delivery, giving new perspectives and context.

- Exploring online collaboration opportunities with other groups (internal and external), building diversity of thinking and reference points. Exploring activities to engage different personalities and individual skillsets such as votes, sketching, metaphors, competition, and gamification.

- Personalisation, giving the student more control over the consumption of material with a HyFlex model of hybrid learning in a flexible structure of pre-recorded deliveries, digital space to socialise work, providing guide rails with supportive content rather than explicit data points.

- Structure, duration, and tone of online sessions - considering the passive versus active balance of content in order to give students a variety of experiences. Break-up screen time with spaces for reflection, a chance to take a break, stretch muscles etc. Give freedom for students to watch content in their own time, whilst explaining the synergies and benefits of group learning.

Institutions and students are mostly not used to these ways of working, so adaption and scaffolding of learning will be necessary. This may ease as hybrid lifestyles become embedded within society, and the benefits to students of a flexible structure with options of modes of attendance according to needs and preference are clear, however we must not lose sight of the benefits of physical design learning spaces.

\section{REFERENCES}

[1] World Economic Forum (2020) Here's how to upgrade education post-pandemic https://www.weforum.org/agenda/2020/12/covid19-education-innovation-outcomes/

[2] Green J. K., Burrow M. S., and Carvalho L. (2020) Designing for transition: supporting teachers and students cope with emergency remote education, Postdigital Science and Education, vol. 2, no. 3, pp. 906-922 [Online]. DOI: 10.1007/s42438-020-00185-6.

[3] Anderson J. (2020) The coronavirus pandemic is reshaping education https://qz.com/1826369/how-coronavirus-is-changing-education/

[4] Sandler R. 2020 Cuomo faces backlash for enlisting billionaires Eric Schmidt and Bill Gates to 'reimagine' NY after reopening (https://www.forbes.com/sites/rachelsandler/2020/05/06/cuomofaces-backlash-for-enlisting-billionaires-eric-schmidt-and-bill-gates-to-reimagine-ny-afterreopening/?sh=7149c2cd5592

[5] Klein N. (2020) How big tech plans to profit from the pandemic https://www.theguardian.com/news/2020/may/13/naomi-klein-how-big-tech-plans-to-profitfrom-coronavirus-pandemic

[6] Klein N. (2007) The Shock Doctrine: The Rise of Disaster Capitalism (Penguin, London)

[7] Microsoft, 2020 https://edudownloads.azureedge.net/msdownloads/MicrosoftEducationReimagined-Paper.pdf

[8] Owen H. in Fleming N. (2020) After Covid, will digital learning be the new normal? https://www.theguardian.com/education/2021/jan/23/after-covid-will-digital-learning-be-thenew-normal

[9] Watters A. (2020) Selling the future of ed-tech http://hackeducation.com

[10] Jones D., Lotz N., and Holden G. (2017) Lurking and learning: Making learning visible in a Virtual Design Studio In: Proceedings of the LearnX Design London 2017 Conference (Pritchard, Gary and Lambert, Nick eds.), London, pp. 176-183. http://oro.open.ac.uk/52977/

[11] Lederman D. (2020) The HyFlex option for instruction if campuses open this fall https://www.insidehighered.com/digital-learning/article/2020/05/13/one-option-deliveringinstruction-if-campuses-open-fall-hyflex 